MITSUBISHI ELECTRIC RESEARCH LABORATORIES

https://www.merl.com

\title{
Coordinating controllers for constrained linear systems by virtual state governors
}

\author{
Di Cairano, S.; Kolmanovsky, I.V. \\ TR2015-011 February 01, 2015
}

\begin{abstract}
Constrained control is often applied to systems with redundant actuation. Often, the usage of a specific group of actuators is to be minimized because of its operating cost and/or undesired side-effects, and, sometimes, controllers for each single actuator may have been previously designed. Thus, for cost or organizational considerations, redesigning the entire control strategy may be impractical. Instead, a coordination scheme for regulating the interaction between the existing controllers while enforcing constraints and minimizing the usage of specific actuators can be developed. We propose a coordination strategy for the case when two groups of actuators, each with a non-modifiable state-feedback controller, are available. By using the maximum constraint admissible set for each controller in closed-loop with the plant, the coordination scheme modulates the action of the assigned controllers and minimizes the usage of the expensive actuators. The proposed control strategy enforces constraints, stabilizes the system, and uses the expensive actuators for finite time and only to avoid constraint violation.
\end{abstract}

IEEE Transactions on Automatic Control

(C) 2015 MERL. This work may not be copied or reproduced in whole or in part for any commercial purpose. Permission to copy in whole or in part without payment of fee is granted for nonprofit educational and research purposes provided that all such whole or partial copies include the following: a notice that such copying is by permission of Mitsubishi Electric Research Laboratories, Inc.; an acknowledgment of the authors and individual contributions to the work; and all applicable portions of the copyright notice. Copying, reproduction, or republishing for any other purpose shall require a license with payment of fee to Mitsubishi Electric Research Laboratories, Inc. All rights reserved. 



\title{
Coordinating controllers for constrained linear systems by virtual state governors
}

\author{
Stefano Di Cairano, Ilya V. Kolmanovsky
}

\begin{abstract}
Constrained control is often applied to systems with redundant actuation. Often, the usage of a specific group of actuators is to be minimized because of its operating cost and/or undesired side-effects, and, sometimes, controllers for each single actuator may have been previously designed. Thus, for cost or organizational considerations, redesigning the entire control strategy may be impractical. Instead, a coordination scheme for regulating the interaction between the existing controllers while enforcing constraints and minimizing the usage of specific actuators can be developed. We propose a coordination strategy for the case when two groups of actuators, each with a non-modifiable state-feedback controller, are available. By using the maximum constraint admissible set for each controller in closed-loop with the plant, the coordination scheme modulates the action of the assigned controllers and minimizes the usage of the "expensive" actuators. The proposed control strategy enforces constraints, stabilizes the system, and uses the expensive actuators for finite time and only to avoid constraint violation.
\end{abstract}

\section{INTRODUCTION}

Today, redundant actuation is being used in several applications. Some examples in automotive include engine idle speed control [1], hybrid electric vehicle energy management [2], and vehicle cornering control by active steering and braking [3]. Aerospace applications include aircraft control by multiple aerodynamic surfaces [4], and spacecraft attitude control [5]. As a specific example of the latter, the International Space Station (ISS) has a cluster of four control moment gyroscopes and 32 attitude control thrusters, and it may rely on attitude control capability from docked service vehicles.

Thus, the coordination of several constrained actuators is a major research area in control. Often, the use of an "expensive" group of actuators has to be minimized, due to high operating cost, or undesired side effects. For instance, in vehicle cornering the use of differential braking shall be avoided when possible due to the longitudinal deceleration that is perceived negatively by the driver, to the noise, and to the increased fuel consumption. In spacecraft attitude control, the use of solar powered momentum exchange actuators is preferred to the use of fuel powered thrusters.

Several techniques have been proposed for designing control systems with redundant actuators, including loop shaping, $\mathcal{H}_{\infty}$ control, and model predictive control, each resulting in a single controller that commands all the actuators. A two stages architecture is used in control allocation [6], which allocates to the physical actuators the virtual commands generated by a higher level controller.

However, in several applications the control strategies and software for the individual actuators may already be available and should be combined without full redesign, for organizational or cost considerations. These cases lead to the approach pursued in this paper, which starts from available non-modifiable controllers for each actuator, and implements a coordination scheme. For instance, in vehicle cornering control [3], the legacy active steering and braking algorithms can be retained, while only the coordination scheme is introduced anew. In the example of the ISS, the actuator configuration may be changing depending on the current service vehicles, and a complete redesign

S. Di Cairano is with Mitsubishi Electric Research Laboratories, Cambridge, MA. E-mail: dicairano@ieee.org.

I.V. Kolmanovsky is with Department of Aerospace Engineering, University of Michigan, Ann Arbor, E-mail: ilya@umich.edu. for each system configuration is clearly impractical. The coordination scheme may also be made responsible for enforcing pointwise-intime state and control constraints. In this way, should the constraints change during the system life cycle or due to particular external conditions, only the coordination scheme is modified. While actuator integration can be achieved by switched control [7], switching between individual actuators often yields suboptimal performance, since only one actuator is active at any time.

In this paper ${ }^{1}$ we consider two groups of actuators, one of which is to be used only when strictly necessary because of higher operating cost, efficiency losses, or undesired side-effects. For each group, a non-modifiable state-feedback controller designed without specifically taking into account control and state constraints is assigned. We develop a coordination strategy that propagates to the assigned controllers "virtual" states, obtained by decomposing the system state based on the constraint admissible sets [9] for the dynamics of the plant in closed loop with each controller. The proposed coordination strategy achieves constraint satisfaction, closed-loop asymptotic stability, enlarged domain of attraction with respect to single actuators, and uses the expensive actuator only when otherwise the constraints would be violated, and only for a finite time.

The proposed control strategy is related to some previously developed approaches, yet it provides unique features. Switching multimode controllers based on constraint admissible sets are proposed in [9], [10], but without actuator coordination. Reference and command governors [11], [12] and extended command governors [13] generate virtual commands for enforcing constraints, but do not coordinate multiple actuators. The proposed control strategy also shares features with reset control systems [14].

The paper is structured as follows. Section II introduces the controller coordination problem, and Section III describes a design that achieves constraint satisfaction. Asymptotic stability and finite time usage of expensive actuators are obtained as described Section IV. A case study in spacecraft attitude control is presented in Section V. Conclusion and extensions are summarized in Section VI.

Notation: $\mathbb{Z}, \mathbb{Z}_{+}, \mathbb{Z}_{0+}$, and $\mathbb{R}, \mathbb{R}_{+}, \mathbb{R}_{0+}$ denote the sets of integers, positive and non-negative integers, and the sets of reals, positive and non-negative reals, respectively. For sets $\mathcal{A}$ and $\mathcal{B}, \mathcal{A} \oplus \mathcal{B}$ is the Minkowski sum, $\operatorname{int}(\mathcal{A})$ is the interior of $\mathcal{A}$, and $\operatorname{Proj}_{x}[\mathcal{A}]$, where $x$ is a vector, is the projection of $\mathcal{A}$ on the subspace of $x .\|\cdot\|$ denotes the Euclidean norm. Relational operators between vectors are intended componentwise, while for matrices denote (semi)definiteness. $[A]_{k}$ where $A$ is a matrix indicates the $k^{t h}$ row, while if $A$ is a vector, indicates the $k^{t h}$ component. 0 indicates the "all-zeros" matrix of appropriate dimensions. For a matrix $P>0, \lambda_{\max }^{P}, \lambda_{\min }^{P}, \kappa(P)$ are the smallest and largest eigenvalue, and their ratio, respectively.

\section{Preliminaries and Problem Definition}

First, we recall some basic definitions and results in invariance and stability that are useful for the subsequent developments.

Definition 1: The set $\mathcal{X} \subseteq \mathbb{R}^{n}$ is positive invariant (PI) for $x(k+$ $1)=f(x(k))$, if for all $x \in \mathcal{X}, f(x) \in \mathcal{X}$.

\footnotetext{
${ }^{1}$ Preliminary results related to this research were presented in [8].
} 
Definition 2: For $x(k+1)=f(x(k)), y(k)=h(x(k))$ subject to $y(k) \in \mathcal{Y}$, the maximum constraint admissible set $\mathcal{O}_{\infty}$ [9] is the largest set such that if $x(k) \in \mathcal{O}_{\infty}$, then $y(t) \in \mathcal{Y}$ for all $t \geq k$.

Note that a system $x(k+1)=\tilde{f}(x(k), u(k))$ controlled by $u(k)=$ $\kappa(x(k))$ subject to $u(k) \in \mathcal{U}$ can be reformulated as in Definition 2 by taking $f(x)=\tilde{f}(x, \kappa(x)), h(x)=\kappa(x), \mathcal{Y} \equiv \mathcal{U}$.

Theorem 1 ([9]): Given an asymptotically stable discrete-time system $x(k+1)=A x(k), y(k)=C x(k)$, where $(A, C)$ is observable, and constraints $y \in \mathcal{Y}$, where $\mathcal{Y}$ is a polytope and $0 \in \operatorname{int}(\mathcal{Y}), \mathcal{O}_{\infty}$ is a polytope, is finitely determined, and $0 \in \operatorname{int}\left(\mathcal{O}_{\infty}\right)$.

Definition 3: A function $\alpha: \mathbb{R}_{0+} \rightarrow \mathbb{R}$ belongs to class $\mathcal{K}$ if it is continuous, strictly increasing and $\alpha(0)=0$. It belongs to class $\mathcal{K}_{\infty}$ if $\alpha \in \mathcal{K}$ and $\alpha(s) \rightarrow \infty$ when $s \rightarrow \infty$.

Definition 4: A function $\mathcal{V}: \mathbb{R}^{n} \rightarrow \mathbb{R}_{0+}$ is a Lyapunov function for $x(k+1)=f(x(k))$ in the PI set $\mathcal{X} \subseteq \mathbb{R}^{n}, 0 \in \operatorname{int}(\mathcal{X})$, if there exist $\bar{\alpha}, \underline{\alpha}, \alpha_{\Delta} \in \mathcal{K}_{\infty}$ such that for all $x \in \mathcal{X}$,

$$
\begin{gathered}
\underline{\alpha}(\|x\|) \leq \mathcal{V}(x) \leq \bar{\alpha}(\|x\|), \\
\Delta \mathcal{V}(x)=\mathcal{V}(f(x))-\mathcal{V}(x) \leq-\alpha_{\Delta}(\|x\|) .
\end{gathered}
$$

Theorem 2 (e.g., [15]): Let $\mathcal{V}: \mathbb{R}^{n} \rightarrow \mathbb{R}_{0+}$ be a Lyapunov function for $x(k+1)=f(x(k))$ in the PI set $\mathcal{X} \subseteq \mathbb{R}^{n}, 0 \in \operatorname{int}(\mathcal{X})$. Then, $x=0$ is asymptotically stable (AS) in $\mathcal{X}$ for $x(k+1)=f(x(k))$.

Next, we formulate the problem addressed in this paper. Consider the discrete-time system

$$
\begin{aligned}
x(k+1) & =A x(k)+B_{1} u_{1}(k)+B_{2} u_{2}(k), \\
y(k) & =C x(k),
\end{aligned}
$$

where $x \in \mathbb{R}^{n}$ is the state vector, $u_{1} \in \mathbb{R}^{m_{1}}, u_{2} \in \mathbb{R}^{m_{2}}$ are input vectors, $y \in \mathbb{R}^{p}$ is the output vector. Even though we often refer to $u_{i}$ as $i^{t h}$ actuator input, $u_{1}, u_{2}$ may each represent a group of inputs (and hence a group of actuators). This observation allows to generalize the developments to the case of $N \in \mathbb{Z}_{+}$actuators.

System (1) is subject to constraints

$$
\begin{aligned}
u_{i} & \in \mathcal{C}_{u, i}, \quad i=\{1,2\}, \\
y & \in \mathcal{C}_{y},
\end{aligned}
$$

where $\mathcal{C}_{y}, \mathcal{C}_{u, i}, i=\{1,2\}$ are given (convex) polyhedra.

The inputs to (1) are generated by two independently designed, non-modifiable controllers

$$
u_{1}=K_{1} x, \quad u_{2}=K_{2} x .
$$

We make the following assumptions.

Assumption 1: $\left(A, B_{i}\right)$ is controllable for $i=1,2$, and $(A, C)$ is observable.

Assumption 2: $K_{i}$ is such that $x(k+1)=\left(A+B_{i} K_{i}\right) x(k)$ is AS for $i=1,2$.

Assumption 3: $\mathcal{C}_{u, i}$ is finitely determined, compact and $0 \in$ $\operatorname{int}\left(\mathcal{C}_{u, i}\right)$ for $i=1,2$. If $\mathcal{C}_{y} \subset \mathbb{R}^{p}, \mathcal{C}_{y}$ is finitely determined, compact and $0 \in \operatorname{int}\left(\mathcal{C}_{y}\right)$.

Note that Assumption 2 does not guarantee that $x(k+1)=$ $A x(k)+B_{1} K_{1} x(k)+B_{2} K_{2} x(k)$ is AS. Neither the pre-assigned controllers (3) guarantee that (2) is satisfied.

In this paper we aim at solving the following problem.

Problem 1: Given (1) and controllers (3) where the gains cannot be modified and the state $x$ is known, design the virtual state governor (VSG) $g: \mathbb{R}^{n} \rightarrow \mathbb{R}^{2 n}$,

$$
g(x)=\left[\begin{array}{l}
g_{1}(x) \\
g_{2}(x)
\end{array}\right]=\left[\begin{array}{l}
x_{1} \\
x_{2}
\end{array}\right]
$$

that provides "virtual states" to (3) resulting in inputs

$$
u_{1}=K_{1} x_{1}, \quad u_{2}=K_{2} x_{2},
$$

such that the closed-loop system

$$
x(k+1)=A x(k)+B_{1} K_{1} x_{1}(k)+B_{2} K_{2} x_{2}(k),
$$

(i) enforces constraints (2), (ii) is asymptotically stable, (iii) $u_{2}(k) \neq 0$ only if (2) cannot be satisfied for every $h \geq k$ by $u_{1}(h)=K_{1} x(h), u_{2}(h)=0$.

At any time $k \in \mathbb{Z}_{0+}$, the control architecture (4), (5) computes $x_{i}(k)=x_{i}, i \in\{1,2\}$, from $x=x(k)$, and assigns $u_{i}(k)=u_{i}$, $i=\{1,2\}$, so that (1) evolves as

$$
x(k+1)=A x(k)+B_{1} K_{1} g_{1}(x(k))+B_{2} K_{2} g_{2}(x(k)) .
$$

Thus (4), (5) is a (nonlinear) static state feedback that modulates the control action of each actuator in order to enforce the constraints, to guarantee asymptotic stability, and to minimize the use of the "expensive" actuator group, $u_{2}$, in the sense that $u_{2} \neq 0$ only when needed for guaranteeing that the constraints are satisfied.

\section{CoOrdination by Virtual State Governor}

First, we construct a design for $g(\cdot)$ in (4) that ensures $(i)$ in Problem 1, and later we specialize it to also ensure $($ ii $),($ iii $)$. We rewrite (6) as

$$
x(k+1)=\left(A+B_{1} K_{1}\right) x_{1}(k)+\left(A+B_{2} K_{2}\right) x_{2}(k),
$$

which can be decomposed into two subsystems

$$
\begin{array}{ll}
\Sigma_{1}: & x_{1}(k+1)=A x_{1}(k)+B_{1} K_{1} x_{1}(k), \\
\Sigma_{2}: & x_{2}(k+1)=A x_{2}(k)+B_{2} K_{2} x_{2}(k) .
\end{array}
$$

Consider first the case when $\mathcal{C}_{y} \equiv \mathbb{R}^{p}$, and let $\mathcal{O}_{\infty}^{i}$ be the maximum constraint-admissible set for system $\Sigma_{i}$, subject to $u_{i} \in \mathcal{C}_{u, i}$, for $i=1,2$. Given $x(k)$, consider the optimization problem,

$$
\begin{array}{rl}
\min _{x_{1}, x_{2}} & J\left(x_{1}, x_{2}\right) \\
\text { s.t. } & x_{1}+x_{2}=x(k) \\
& x_{i}(k) \in \mathcal{O}_{\infty}^{i}, \quad i=1,2,
\end{array}
$$

where $J: \mathbb{R}^{n} \times \mathbb{R}^{n} \rightarrow \mathbb{R}_{0+}$ is a cost function. Constraint (10b) decomposes $x$ in two vectors, $x=x_{1}+x_{2}$, each to be provided to one of the predefined controllers, i.e., each used for feedback through one of the actuators. Cost function (10a) may be defined to minimize the use of the expensive actuator, while (10c) ensures that the decomposition (10b) satisfies the constraints at every future time instant. The effect of (10b), (10c) is to decompose the state vector $x$ into $x_{1} \in \mathcal{O}_{\infty}^{1}, x_{2} \in \mathcal{O}_{\infty}^{2}$ such that $x_{1}+x_{2}=x$. Among all admissible decompositions, the one that minimizes the value of the cost function (10a) is selected.

Let $\left[x_{1}^{*}(x(k))^{\prime} x_{2}^{*}(x(k))^{\prime}\right]^{\prime}$ be the optimizer of (10), and define

$$
g(x(k))=\left[\begin{array}{l}
x_{1}^{*}(x(k)) \\
x_{2}^{*}(x(k))
\end{array}\right],
$$

so that $x_{i}(k)=x_{i}^{*}(x(k))=g_{i}(x(k))$, and indeed if $x_{1}^{*}(x(k))=$ $x(k), u_{2}(k)=0$. Let $\mathcal{X}_{\mathrm{f}}$ be the set of states such that (10) is feasible, i.e., $\mathcal{X}_{\mathrm{f}} \equiv\left\{x \in \mathbb{R}^{n}: \exists x_{i} \in \mathcal{O}_{\infty}^{i}, i \in\{1,2\}, x_{1}+x_{2}=x\right\}$.

Theorem 3: For all $x(k) \in\left(\mathcal{O}_{\infty}^{1} \oplus \mathcal{O}_{\infty}^{2}\right),(10)$ admits a feasible solution. For (7), if (10) is feasible at $k \in \mathbb{Z}_{0+}$, then it is feasible for all $t \in \mathbb{Z}_{0+}, t \geq k$, i.e., $\mathcal{X}_{\mathrm{f}}$ is PI for (7), (11).

Proof: If $\left(x_{1}, x_{2}\right) \in \mathbb{R}^{n} \times \mathbb{R}^{n}$ is feasible for (10), then $x_{i} \in \mathcal{O}_{\infty}^{i}$, $i=\{1,2\}$, and $x_{1}+x_{2}=x$. Hence, $\mathcal{X}_{f} \equiv\left(\mathcal{O}_{\infty}^{1} \oplus \mathcal{O}_{\infty}^{2}\right)$. Assume that $x(k)$ is feasible and let $x_{1}(k), x_{2}(k)$ be a solution of (10). Then, (7) evolves according to (8). Thus, $\tilde{x}_{i}(k+1)=\left(A+B_{i} K_{i}\right) x_{i}(k)$, $i \in\{1,2\}$, are such that $x(k+1)=\tilde{x}_{1}(k+1)+\tilde{x}_{2}(k+1)$. Since $x_{i}(k) \in \mathcal{O}_{\infty}^{i},\left(A+B_{i} K_{i}\right)^{j} x_{i}(k) \in \mathcal{O}_{\infty}^{i}$ for any $j \in \mathbb{Z}_{0+}$, and hence $x_{i}(k+1) \in \mathcal{O}_{\infty}^{i}$, for any $i \in\{1,2\}$. Hence, the decomposition 
$\tilde{x}_{i}(k+1)=\left(A+B_{i} K_{i}\right) x_{i}(k), i \in\{1,2\}$ is feasible. Then, the claim follows by induction.

From Theorem 3 the VSG-controlled system has larger domain of attraction $\left(\mathcal{O}_{\infty}^{1} \oplus \mathcal{O}_{\infty}^{2}\right)$ than the ones achieved when the single actuators are used $\left(\mathcal{O}_{\infty}^{i}, i=1,2\right)$, and when a switching control law based on $\mathcal{O}_{\infty}^{i}$ is used $\left(\mathcal{O}_{\infty}^{1} \cup \mathcal{O}_{\infty}^{2}\right)$.

When $\mathcal{C}_{y} \subset \mathbb{R}^{p}$ the output constraints couple the subsystems requiring a slightly more complex decomposition in (8). Let $\mathcal{C}_{y} \equiv$ $\left\{x \in \mathbb{R}^{n}: H C x \leq h\right\}$, where $H \in \mathbb{R}^{\ell \times p}, h \in \mathbb{R}^{\ell}$, and for $i=1,2$ introduce the additional state vector $\varepsilon_{i} \in \mathbb{R}^{\ell}$, representing the constraint range allocated to $\Sigma_{i}$. Then, compute the maximum constraint admissible set $\overline{\mathcal{O}}_{\infty}^{i}$ for

$$
\begin{aligned}
x_{i}(k+1) & =\left(A+B_{i} K_{i}\right) x_{i}(k), \\
\varepsilon_{i}(k+1) & =\varepsilon_{i}(k), \\
H C x_{i}(k) & \leq \phi+\varepsilon_{i}(k), \\
0 & \leq \varepsilon_{i}(k) \leq h-\phi,
\end{aligned}
$$

for $i \in\{1,2\}$, where $\phi \in \mathbb{R}^{\ell}$, such that $[\phi]_{j} \in\left(0,[h]_{j}\right)$, for all $j=1, \ldots \ell$ is a fixed constant vector, finite yet possibly arbitrarily small, and $0 \in \operatorname{int}(\mathcal{H}), \mathcal{H}=\left\{x \in \mathbb{R}^{n}: H C x \leq \phi\right\}$. Note that $\overline{\mathcal{O}}_{\infty}^{i}$ is defined on $\left(x_{i}, \varepsilon_{i}\right) \in \mathbb{R}^{n+\ell}$. Given $\varepsilon_{i}, \mathcal{O}_{\infty}^{i}\left(\varepsilon_{i}\right) \equiv\left\{x_{i} \in \mathbb{R}^{n}\right.$ : $\left.\left(x_{i}, \varepsilon_{i}\right) \in \overline{\mathcal{O}}_{\infty}^{i}\right\}$, i.e., the cross-section of $\overline{\mathcal{O}}_{\infty}^{i}$ for given $\varepsilon_{i}$.

Theorem 1 does not apply to (12), since (12b) is not AS. However, under appropriate assumptions, a similar result holds.

Lemma 1: Given $x(k+1)=A x(k), y(k)=C x(k)$, let $A$ be strictly Schur, and $(A, C)$ observable. Let the output constraint set $\mathcal{Y} \equiv\left\{y \in \mathbb{R}^{\ell}: H y \leq \phi\right\}$, where $H \in \mathbb{R}^{\ell \times p}, \phi \in \mathbb{R}^{\ell}$, be compact and $0 \in \operatorname{int}(\mathcal{Y})$. For every finite $\varepsilon_{\max } \in \mathbb{R}_{+}$, the maximum constraint admissible set of (12), $\overline{\mathcal{O}}_{\infty} \equiv\left\{(x, \varepsilon) \in \mathbb{R}^{n+\ell}: H C A^{k} x \leq \phi+\right.$ $\left.\varepsilon, 0 \leq \varepsilon \leq \varepsilon_{\max }, k \in \mathbb{Z}_{0+}\right\}$, is compact and finitely determined.

Proof: Consider $\varepsilon>0$, and $\mathcal{Y}_{\varepsilon} \equiv\left\{y \in \mathbb{R}^{p}: H y \leq \phi+\varepsilon\right\}$. For $n \in \mathbb{Z}_{+}$, let $\overline{\mathcal{O}}_{n} \equiv\left\{(x, \varepsilon) \in \mathbb{R}^{n+\ell}: C A^{k} x \in \mathcal{Y}_{\varepsilon}, 0 \leq \varepsilon \leq\right.$ $\left.\varepsilon_{\text {max }}, k=0, \ldots, n-1\right\}$. Clearly, $\overline{\mathcal{O}}_{\infty} \subset \overline{\mathcal{O}}_{n}$. We prove that: $(a) \overline{\mathcal{Y}}_{\varepsilon}$ is compact, $(b) \overline{\mathcal{O}}_{\infty}$ is bounded, $(c) \overline{\mathcal{O}}_{\infty}$ is finitely determined and closed, and hence compact.

(a) Clearly, $\mathcal{Y}_{\varepsilon}$ is closed, so we need to show that $\mathcal{Y}_{\varepsilon}$ is bounded. If $\mathcal{Y}_{\varepsilon}$ is unbounded, by convexity, compactness of the unit sphere, and $0 \in \mathcal{Y}_{\varepsilon}$ there must exist $e \in \mathbb{R}^{p}$ with $\|e\|=1$ such that $\lambda e \in \mathcal{Y}_{\varepsilon}$, for all $\lambda \geq 0$. Since $\mathcal{Y}$ is compact, there exists $\bar{\lambda}>0$ such that for all $\lambda>\bar{\lambda}$, $\lambda e \notin \mathcal{Y}$. Thus, there exists $\bar{\imath} \in \mathbb{Z}_{+}, 1 \leq \bar{\imath} \leq \ell$, such that $[H]_{\bar{\imath}} \lambda e>[h]_{\bar{\imath}}$ for all $\lambda>\bar{\lambda}$. Since $0 \in \operatorname{int}(\mathcal{Y}),[h]_{\bar{\imath}}>0$ and $[H]_{\bar{\imath}} e>0$. Then, there exists $\hat{\lambda}>0$ such that $[H]_{\bar{\imath}} \lambda e>[h]_{\bar{\imath}}+\varepsilon_{\max }$ for all $\lambda>\hat{\lambda}$, which is contradicting $\lambda e \in \mathcal{Y}_{\varepsilon}$ for all $\lambda>0$.

(b) By assumption, the observability matrix $\Gamma \in \mathbb{R}^{n p \times n}=$ $\left[C^{\prime}(C A)^{\prime} \ldots\left(C A^{n-1}\right)^{\prime}\right]$ has rank $n$, thus $\Gamma^{\prime} \Gamma>0$. Hence, $\overline{\mathcal{O}}_{n}=$ $\left\{(x, \varepsilon): \Gamma x \in \mathcal{Y}_{\varepsilon} \times \ldots \times \mathcal{Y}_{\varepsilon} \subset \mathbb{R}^{n p}, 0 \leq \varepsilon \leq \varepsilon_{\max }\right\}$, is bounded since $\mathcal{Y}_{\varepsilon}$ is bounded for any $0 \leq \varepsilon \leq \varepsilon_{\max }$. Hence, $\overline{\mathcal{O}}_{\infty} \subset \overline{\mathcal{O}}_{n}$ is bounded.

(c) By assumption, $\lim _{k \rightarrow \infty}\left\|A^{k}\right\|=0$. Since $\overline{\mathcal{O}}_{k}$ is bounded for $k \geq n$, there exists $k^{*}$ such that for all $x \in \overline{\mathcal{O}}_{k^{*}}, H C A^{k} x<\phi$ for all $k \geq k^{*}$. Thus, $H C A^{k} x \leq \phi+\varepsilon, 0 \leq \varepsilon \leq \varepsilon_{\max }$, for all $k=0, \ldots, k^{*}$ implies $H C A^{k} x \leq \phi+\varepsilon$, for all $k \geq k^{*}$. Hence $\overline{\mathcal{O}}_{k^{*}}=\overline{\mathcal{O}}_{\infty}$, and since $\overline{\mathcal{O}}_{k^{*}}$ is the intersection of a finite number of closed sets, $\overline{\mathcal{O}}_{\infty}$ is finitely determined, closed and hence compact.

Given $x(k)$, consider the optimization problem

$$
\begin{array}{rl}
\min _{x_{1}, x_{2}, \varepsilon_{1}, \varepsilon_{2}} & J\left(x_{1}, x_{2}, \varepsilon_{1}, \varepsilon_{2}\right) \\
\text { s.t. } & x_{1}+x_{2}=x(k) \\
& x_{i} \in \mathcal{O}_{\infty}^{i}\left(\varepsilon_{i}\right), i=1,2 \\
& \varepsilon_{1}+\varepsilon_{2} \leq h-2 \phi
\end{array}
$$

let $\left[x_{1}^{*}(x(k))^{\prime} x_{2}^{*}(x(k))^{\prime} \varepsilon_{1}^{*}(x(k))^{\prime} \varepsilon_{2}^{*}(x(k))^{\prime}\right]^{\prime}$ be the optimizer, and define $g(x(k))$ by (11), where $\varepsilon_{1}^{*}, \varepsilon_{2}^{*}$ do not (explicitly) appear. By (13), the controller decomposes not only the state between the different subsystems, but also the constraint bounds. Constraint (13c) ensures $H C x_{i}(k) \leq \phi+\varepsilon_{i}(k), i \in\{1,2\}$, and, by (13b), (13d), $H C x(k)=H C\left(x_{1}(k)+x_{2}(k)\right) \leq \varepsilon_{1}(k)+\varepsilon_{2}(k)+2 \phi \leq h$. If (13) is used in place of (10), the set of feasible states is $\mathcal{X}_{\mathrm{f}} \equiv\left\{x \in \mathbb{R}^{n}\right.$ : $\left.\exists\left(x_{i}, \varepsilon_{i}\right) \in \overline{\mathcal{O}}_{\infty}^{i}, i \in\{1,2\}, x_{1}+x_{2}=x, \varepsilon_{1}+\varepsilon_{2} \leq h-2 \phi\right\}$.

Theorem 4: Under the assumptions of Lemma $1, \mathcal{X}_{\mathrm{f}}$ is convex, compact and finitely determined. For (7), if (13) is feasible at $k \in$ $\mathbb{Z}_{0+}$, then the constraints are satisfied at any time instant $t \geq k$, $t \in \mathbb{Z}_{0+}$, i.e., $\mathcal{X}_{\mathrm{f}}$ is PI for (7), (11).

Proof: The sets $\overline{\mathcal{O}}_{\infty}^{i}, i \in\{1,2\}$ are convex, and so are the sets defined by (13b), (13d). Hence, $\mathcal{X}_{\mathrm{f}}$ is convex. By Lemma 1 , $\overline{\mathcal{O}}_{\infty}^{i}, i \in\{1,2\}$ is compact and finitely determined. Since $\mathcal{X}_{\mathrm{f}}$ is the intersection of the sets defined by (13b), (13c), (13d), which are compact and finitely determined, so is $\mathcal{X}_{\mathrm{f}}$. Let (13) be feasible at time $k \in \mathbb{Z}_{0+}$ for $x(k)$, and let $x_{i}(k) \in \mathbb{R}^{n}, \varepsilon_{i}(k) \in \mathbb{R}^{\ell}, i \in\{1,2\}$ be selected. Then, at time $k+1,\left(\left(A+B_{i} K_{i}\right) x_{i}(k), \varepsilon_{i}(k)\right), i \in\{1,2\}$ is a feasible solution for (13), since $\left(x_{i}(k), \varepsilon_{i}(k)\right) \in \overline{\mathcal{O}}_{\infty}^{i}$ implies $\left(\left(A+B_{i} K_{i}\right) x_{i}(k), \varepsilon_{i}(k)\right) \in \overline{\mathcal{O}}_{\infty}^{i}$ by invariance of $\overline{\mathcal{O}}_{\infty}^{i}$. Then, the claim follows by induction.

Theorems 3 and 4 ensure that (11) and (10) or (13) satisfy $(i)$ in Problem 1. Next, we show that (ii) and (iii) can be obtained through a proper choice of $J$ in (10a) or (13a).

\section{Closed-Loop Stability}

Consider the Lyapunov functions $\mathcal{V}_{i}\left(x_{i}\right)=x_{i}^{\prime} P_{i} x_{i}$ for $\Sigma_{i}, i=$ 1,2 , where $P_{i}>0$ is such that $\left(A+B_{i} K_{i}\right)^{\prime} P_{i}\left(A+B_{i} K_{i}\right)-P_{i}=$ $-Q_{i}$, for some $Q_{i}>0$, for $i \in\{1,2\}$. In what follows, $\mu_{i}=$ $\left\|A+B_{i} K_{i}\right\|$ is the induced norm of the state update matrix of $\Sigma_{i}$, for $i \in\{1,2\}$.

In order to achieve $(i i)$ and $($ iii $)$ in Problem 1 we propose to use as the cost function in (10), (13)

$$
J\left(x_{1}, x_{2}\right)=J\left(x_{2}\right)=\left\|P_{2}^{1 / 2} x_{2}\right\|^{2}=x_{2}^{\prime} P_{2} x_{2},
$$

where $P_{2}$ is such that $x_{2}^{\prime} P_{2} x_{2}$ is a Lyapunov function for $x_{2}(k+$ $1)=\left(A+B_{2} K_{2}\right) x_{2}(k)$. By (14), (11) selects $x_{2}$ that belongs to the minimum achievable level set of a Lyapunov function for $\Sigma_{2}$. Thus, by (14), (11) minimizes the value of the Lyapunov function of the closed-loop system $x_{2}(k+1)=\left(A+B_{2} K_{2}\right) x_{2}(k)$, that is the closed-loop subsystem associated with the expensive actuator, which could be regarded as a measure of "energy" in $\Sigma_{2}$.

Remark 1: With (14), (10) and (13) result in convex quadratic programs, since for (1), (2), $\mathcal{O}_{\infty}^{i}, \overline{\mathcal{O}}_{\infty}^{i}$ are polyhedra for $i=1,2$. Several efficient algorithms for solving QP exist (see e.g., [16] and references therein). For these cases, the VSG control law (11) can also be explicitly computed by multiparametric programming resulting in a piecewise affine control law [17]. Thus, online optimization can be avoided. The complexity of the VSG control law can be further reduced by storing only $g_{1}(x)$, from which $g_{2}(x)$ can be reconstructed, and consequently merging the regions of the control law with equal $g_{1}(x)$, see, e.g., [18].

Next, we show that, (11), with (10) (or (13)) and (14) achieves (ii) of Problem 1, i.e., the origin is asymptotically stable for the closedloop system. In what follows we explicitly refer to (10), while it is understood that (13) is to be used when output constraints are present.

Given the Lyapunov functions $\mathcal{V}_{i}\left(x_{i}\right)$ for $\Sigma_{i}, i=1,2$, we prove that there exists $\gamma>0$ such that $\mathcal{V}(x)=\mathcal{V}_{1}\left(x_{1}\right)+\gamma \mathcal{V}\left(x_{2}\right)$ is a Lyapunov function for $x(k+1)=A x(k)+B_{1} K_{1} g_{1}(x(k))+$ $B_{2} K_{2} g_{2}(x(k))$, for $g_{i}(x(k)), i=1,2$, defined by (11).

Assumption 4: Either at least one of $\mathcal{O}_{\infty}^{i}, i=1,2$, is bounded or the minimum of (10) is bounded at $k=0$. 
Lemma 2: Along the closed-loop trajectories of (7) with $g(\cdot)$ defined by (11), (14), there exists $c_{i}>0, i=1,2$, such that $\left\|x_{i}(k)\right\| \leq c_{i}\|x(k)\|, i=1,2$.

Proof: We prove only the cases when the minimum of (10) is bounded at $k=0$, and $\mathcal{O}_{\infty}^{2}$ is bounded. The proof for when $\mathcal{O}_{\infty}^{1}$ is bounded is similar. Since $0 \in \operatorname{int}\left(\mathcal{O}_{\infty}^{i}\right), i=1,2$, there exists $\rho>0$ such that $\left\{x_{1} \in \mathbb{R}^{n}:\left\|x_{1}\right\| \leq \rho\right\} \subseteq \mathcal{O}_{\infty}^{1}$, and since $x_{2}(k)$ is selected by (10), (14), whenever $\|x(k)\| \leq \rho$, we have $x_{1}(k)=x(k)$, $x_{2}(k)=0$.

Let $\|x(k)\| \geq \rho$. Suppose that the minimum of (10) is bounded at $k=0$, i.e., $x_{2}(0)^{\prime} P_{2} x_{2}(0) \leq \tilde{\beta}$. Since $\mathcal{V}_{2}\left(x_{2}\right)=x_{2}^{\prime} P_{2} x_{2}$ is a Lyapunov function for $\Sigma_{2}, \mathcal{V}_{2}\left(\left(A+B_{2} K_{2}\right) x_{2}(0)\right) \leq \tilde{\beta}$, and given that for all $k \in \mathbb{Z}_{0+}, x_{2}(k)$ is the optimum of (10) with $\left(A+B_{2} K_{2}\right) x_{2}(k)$ being a feasible solution, $\mathcal{V}_{2}\left(x_{2}(k+1)\right) \leq \mathcal{V}_{2}\left(x_{2}(k)\right) \leq \tilde{\beta}$. Since $\mathcal{V}_{2}\left(x_{2}\right)$ is a Lyapunov function, $\underline{\alpha}_{2}\left(\left\|x_{2}(k)\right\|\right) \leq \mathcal{V}\left(x_{2}(k)\right) \leq$ $\mathcal{V}\left(x_{2}(0)\right) \leq \tilde{\beta}$. Given that $\tilde{\beta}$ is finite and $\underline{\alpha}_{2} \in \mathcal{K}_{\infty}$, for some $\beta>0$, $\left\|x_{2}(k)\right\| \leq \beta$ for all $k \in \mathbb{Z}_{0+}$.

Instead of the minimum of (10) being bounded at $k=0$, suppose $\mathcal{O}_{\infty}^{2}$ is bounded. Then, along any feasible trajectory there exists $\beta>$ 0 such that $\left\|x_{2}(k)\right\| \leq \beta$ for all $k \in \mathbb{Z}_{0+}$.

Thus, for all $k \in \mathbb{Z}_{0+}$, when $\|x(k)\| \geq \rho,\left\|x_{1}(k)\right\| \leq\|x(k)\|+$ $\left\|x_{2}(k)\right\| \leq\|x(k)\|+\beta \leq\|x(k)\|+\beta \frac{\|x(k)\|}{\rho}=\left(1+\frac{\beta}{\rho}\right)\|x(k)\|$. Since $\left\|x_{2}(k)\right\| \leq\|x(k)\|+\left\|x_{1}(k)\right\|, c_{1}=\left(1+\frac{\beta}{\rho}\right)$ and $c_{2}=\left(2+\frac{\beta}{\rho}\right)$ satisfy the statement, including when $\|x(k)\| \leq \rho$.

Lemma 3: Let $x_{i}(k) \in \mathcal{O}_{\infty}^{i}, i=1,2$ be given, let $\tilde{x}_{2}(k+$ $1)=\left(A+B_{2} K_{2}\right) x_{2}(k)$, and let $x_{2}(k+1)$ be obtained by solving (10), (14). There exists $c \in \mathbb{R}_{+}$such that $\| x_{2}(k+1)-$ $\tilde{x}_{2}(k+1)\|\leq c\| x_{2}(k) \|$.

Proof: By optimality of $x_{2}(k+1)$ and feasibility of $\tilde{x}_{2}(k+1)$, we have that $x_{2}(k+1)^{\prime} P_{2} x_{2}(k+1) \leq \tilde{x}_{2}(k+1)^{\prime} P_{2} \tilde{x}_{2}(k+1) \leq$ $\lambda_{\max }^{P_{2}}\left\|\tilde{x}_{2}(k+1)\right\|^{2}$. Since $x_{2}(k+1)^{\prime} P_{2} x_{2}(k+1) \geq \lambda_{\min }^{P_{2}} \| x_{2}(k+$ 1) $\|^{2}$, it holds that $\left\|x_{2}(k+1)\right\| \leq \sqrt{\kappa\left(P_{2}\right)}\left\|\tilde{x}_{2}(k+1)\right\|=\tilde{c} \| \tilde{x}_{2}(k+$ 1) $\|$. Thus, $\left\|x_{2}(k+1)-\tilde{x}_{2}(k+1)\right\| \leq\left\|x_{2}(k+1)\right\|+\left\|\tilde{x}_{2}(k+1)\right\| \leq$ $(\tilde{c}+1)\left\|\tilde{x}_{2}(k+1)\right\| \leq(\tilde{c}+1) \mu_{2}\left\|x_{2}(k)\right\|=c\left\|x_{2}(k)\right\|$.

Theorem 5: Let $\mathcal{V}_{i}\left(x_{i}\right)=x_{i}^{\prime} P_{i} x_{i}$ be a Lyapunov function for $x_{i}(k+1)=\left(A+B_{i} K_{i}\right) x_{i}(k), i \in\{1,2\}$. Then, there exists $\gamma \in \mathbb{R}_{+}$ such that $\mathcal{V}(x)=\mathcal{V}_{1}\left(x_{1}\right)+\gamma \mathcal{V}_{2}\left(x_{2}\right)$ is a Lyapunov function within $\mathcal{X}_{\mathrm{f}}$ for (7) in closed-loop with $g(x(k))$ defined by (11), (10), (14).

Proof: First note that because of Theorem 3/ Theorem 4, $\mathcal{X}_{\mathrm{f}}$ is PI for the closed-loop dynamics. In order to prove that $\mathcal{V}(x)$ is a Lyapunov function, we first need to prove that there exists $\bar{\alpha}, \underline{\alpha} \in \mathcal{K}_{\infty}$ such that $\underline{\alpha}(\|x\|) \leq \mathcal{V}(x) \leq \bar{\alpha}(\|x\|)$. For any $\gamma>0, \mathcal{V}(x) \leq$ $\bar{\alpha}_{1}\left(\left\|x_{1}\right\|\right)+\gamma \bar{\alpha}_{2}\left(\left\|x_{2}\right\|\right)$, and, by Lemma $2, \mathcal{V}(x) \leq \bar{\alpha}_{1}\left(c_{1}\|x\|\right)+$ $\gamma \bar{\alpha}_{2}\left(c_{2}\|x\|\right)$. Hence, $\bar{\alpha}(\|x\|)=\bar{\alpha}_{1}\left(c_{1}\|x\|\right)+\gamma \bar{\alpha}_{2}\left(c_{2}\|x\|\right)$ is an upper bound on $\mathcal{V}(x)$, and indeed $\bar{\alpha}(\|x\|) \in \mathcal{K}_{\infty}$ since $\gamma>0$, and $\bar{\alpha}_{1}(\|x\|), \bar{\alpha}_{2}(\|x\|) \in \mathcal{K}_{\infty}$. By the definition of $\mathcal{V}(x)$, for any $\gamma>0$, $\mathcal{V}(x) \geq \underline{\alpha}_{1}\left(\left\|x_{1}\right\|\right)+\gamma \underline{\alpha}_{2}\left(\left\|x_{2}\right\|\right)$. Consider the case $\left\|x_{2}\right\| \leq\left\|x_{1}\right\|$. Then, $\underline{\alpha}_{1}\left(\left\|x_{1}\right\|\right)+\gamma \underline{\alpha}_{2}\left(\left\|x_{2}\right\|\right) \geq \underline{\alpha}_{1}\left(\left\|x_{1}\right\|\right) \geq \underline{\alpha}_{1}\left(\frac{1}{2}\left\|x_{1}\right\|+\frac{1}{2}\left\|x_{2}\right\|\right) \geq$ $\underline{\alpha}_{1}\left(\frac{1}{2}\|x\|\right)$. Consider the case $\left\|x_{1}\right\| \leq\left\|x_{2}\right\|$. Then, $\underline{\alpha}_{1}\left(\left\|x_{1}\right\|\right)+$ $\gamma \underline{\alpha}_{2}\left(\left\|x_{2}\right\|\right) \geq \gamma \underline{\alpha}_{2}\left(\left\|x_{2}\right\|\right) \geq \gamma \underline{\alpha}_{2}\left(\frac{1}{2}\left\|x_{1}\right\|+\frac{1}{2}\left\|x_{2}\right\|\right) \geq \gamma \underline{\alpha}_{2}\left(\frac{1}{2}\|x\|\right)$. Thus, $\mathcal{V}(x) \geq \min \left(\underline{\alpha}_{1}\left(\frac{1}{2}\|x\|\right), \gamma \underline{\alpha}_{2}\left(\frac{1}{2}\|x\|\right)\right)=\underline{\alpha}(\|x\|)$ and for any $\gamma>0, \underline{\alpha}(\|x\|) \in \mathcal{K}_{\infty}$.

Next, to prove that there exists $\alpha_{\Delta}(\|x\|) \in \mathcal{K}_{\infty}$ such that $\Delta \mathcal{V}(x) \leq-\alpha_{\Delta}(\|x\|)$. Let $x, x_{i} \in \mathbb{R}^{n}, i=1,2$, be given, where $x=x_{1}+x_{2}$, and consider the evolution as composed of two stages: ( $i$ ) continuous evolution of (9) resulting in $\tilde{x}_{i}=\left(A+B_{i} K_{i}\right) x_{i}, i=$ $1,2,($ ii $)$ reset of the subsystem states ${ }^{2}$ by (11), i.e, $x_{i}^{+}=g_{i}\left(\tilde{x}_{1}, \tilde{x}_{2}\right)$, $i=1,2$, where $x^{+}=x_{1}^{+}+x_{2}^{+}=\tilde{x}_{1}+\tilde{x}_{2}$, by (10b). Thus,

$$
\Delta \mathcal{V}(x)=\Delta \tilde{\mathcal{V}}\left(x_{1}, x_{2}, \tilde{x}_{1}, \tilde{x}_{2}\right)+\phi\left(\tilde{x}_{1}, \tilde{x}_{2}, x_{1}^{+}, x_{2}^{+}\right)
$$

\footnotetext{
${ }^{2}$ Since the reset does not change the actual system state, and at every step the closed-loop dynamics evolve by a continuous evolution and a reset, we can arbitrarily choose which of the two actions is considered first.
}

where $\Delta \tilde{\mathcal{V}}\left(x_{1}, x_{2}, \tilde{x}_{1}, \tilde{x}_{2}\right)$ is the change of $\mathcal{V}(x)$ during the continuous execution of the subsystems, while $\phi\left(\tilde{x}_{1}, \tilde{x}_{2}, x_{1}, x_{2}\right)$ is the change of $\mathcal{V}(x)$ caused by the reset due to (11).

Since $\mathcal{V}_{i}(x), i=1,2$, is a Lyapunov function for (9), $\tilde{x}_{i}^{\prime} P_{i} \tilde{x}_{i}-$ $x_{i}^{\prime} P_{i} x_{i}=-x_{i}^{\prime} Q_{i} x_{i}, Q_{i}>0, i=1,2$. Thus in (15), for any $\gamma>0$, whenever $\left\|x_{1}\right\|+\left\|x_{2}\right\|>0$,

$$
\Delta \tilde{\mathcal{V}}\left(x_{1}, x_{2}, \tilde{x}_{1}, \tilde{x}_{2}\right)=-x_{1}^{\prime} Q_{1} x_{1}-\gamma x_{2}^{\prime} Q_{2} x_{2}<0 .
$$

In $(15)$, the term related to the reset is

$$
\begin{aligned}
\phi\left(\tilde{x}_{1}, \tilde{x}_{2}, x_{1}^{+}, x_{2}^{+}\right)=\left(\mathcal{V}_{1}\left(x_{1}^{+}\right)\right. & \left.-\mathcal{V}_{1}\left(\tilde{x}_{1}^{+}\right)\right)+ \\
& \gamma\left(\mathcal{V}_{2}\left(x_{2}^{+}\right)-\mathcal{V}_{2}\left(\tilde{x}_{2}^{+}\right)\right) .
\end{aligned}
$$

Since $\tilde{x}_{i}, i=1,2$ is feasible for (10) with cost function (14), while $x_{i}^{+}, i=1,2$ is the optimizer, $\mathcal{V}_{2}\left(x_{2}^{+}\right)-\mathcal{V}_{2}\left(\tilde{x}_{2}\right) \leq 0$. Thus, we have a bound on (15),

$$
\begin{aligned}
& -\Delta \mathcal{V}(x) \geq \varphi\left(x_{1}, x_{2}, \tilde{x}_{1}, x_{1}^{+}\right)= \\
& x_{1}^{\prime} Q_{1} x_{1}+\gamma x_{2}^{\prime} Q_{2} x_{2}-\left(\mathcal{V}_{1}\left(x_{1}^{+}\right)-\mathcal{V}_{1}\left(\tilde{x}_{1}\right)\right) .
\end{aligned}
$$

Next, we show $\varphi$ is lower bounded by a class- $\mathcal{K}$ function. Let us call $\delta=x_{1}^{+}-\tilde{x}_{1}$, then

$$
\mathcal{V}_{1}\left(x_{1}^{+}\right)-\mathcal{V}_{1}\left(\tilde{x}_{1}\right)=\delta^{\prime} P_{1} \delta+2 \delta^{\prime} P_{1}\left(A+B_{1} K_{1}\right) x_{1} .
$$

We have $x_{1}^{\prime} Q_{1} x_{1}+\gamma x_{2}^{\prime} Q_{2} x_{2} \geq\left\|x_{1}\right\|^{2} \lambda_{\text {min }}^{Q_{1}}+\gamma\left\|x_{2}\right\|^{2} \lambda_{\text {min }}^{Q_{2}}$, and

$$
\delta^{\prime} P_{1} \delta+2 \delta P_{1}\left(A+B_{1} K_{1}\right) x_{1} \leq\|\delta\|^{2} \lambda_{\max }^{P_{1}}+2 \mu_{1} \lambda_{\max }^{P_{1}}\|\delta\|\left\|x_{1}\right\| .
$$

Hence, $\varphi_{1}\left(x_{1}, x_{2}, \delta\right) \leq \varphi\left(x_{1}, x_{2}, \delta\right)$ where

$$
\begin{aligned}
\varphi_{1}\left(x_{1}, x_{2}, \delta\right)= & \left\|x_{1}\right\|^{2} \lambda_{\min }^{Q_{1}}+\gamma\left\|x_{2}\right\|^{2} \lambda_{\min }^{Q_{2}} \\
& -\left(\|\delta\|^{2} \lambda_{\max }^{P_{1}}+2 \mu_{1} \lambda_{\max }^{P_{1}}\|\delta\|\left\|x_{1}\right\|\right) .
\end{aligned}
$$

It also follows that $\varphi_{2}\left(x_{1}, x_{2}\right) \leq \varphi_{1}\left(x_{1}, x_{2}, \delta\right)$, where

$$
\begin{aligned}
\varphi_{2}\left(x_{1}, x_{2}\right)= & \left\|x_{1}\right\|^{2} \lambda_{\min }^{Q_{1}}+\gamma\left\|x_{2}\right\|^{2} \lambda_{\min }^{Q_{2}} \\
& -c^{2}\left\|x_{2}\right\|^{2} \lambda_{\max }^{P_{1}}-2 c \mu_{1} \lambda_{\max }^{P_{1}}\left\|x_{2}\right\|\left\|x_{1}\right\|,
\end{aligned}
$$

because $-\left(x_{2}^{+}-\tilde{x}_{2}\right)=x_{1}^{+}-\tilde{x}_{1}=\delta$ and hence $\|\delta\| \leq c\left\|x_{2}\right\|$, by Lemma 3. Since for any $a, b \in \mathbb{R}, q \in \mathbb{R}_{+},|a b| \leq \frac{a^{2}}{2 q}+\frac{b^{2} q}{2}$,

$$
\begin{aligned}
\varphi_{2}\left(x_{1}, x_{2}\right) \geq & \left\|x_{1}\right\|^{2}\left(\lambda_{\min }^{Q_{1}}-\frac{2 c \mu_{1} \lambda_{\max }^{P_{1}}}{2 q}\right) \\
& +\left\|x_{2}\right\|^{2}\left(\gamma \lambda_{\min }^{Q_{2}}-c^{2} \lambda_{\max }^{P_{1}}-\frac{2 c \mu_{1} \lambda_{\max }^{P_{1}} q}{2}\right),
\end{aligned}
$$

where, $q, \gamma \in \mathbb{R}_{+}$are arbitrary. Thus, we can find $q>0$ such that $\left(\lambda_{\min }^{Q_{1}}-\frac{2 c \mu_{1} \lambda_{\max }^{P_{1}}}{2 q}\right)=\beta_{1}>0$, and $\gamma>0$ such that $\left(\gamma \lambda_{\min }^{Q_{2}}-c^{2} \lambda_{\max }^{P_{1}}-\frac{2 c \mu_{1} \lambda_{\max }^{P_{1}} q}{2}\right)=\beta_{2}>0$. By defining $\beta=$ $\min \left\{\beta_{1}, \beta_{2}\right\}$,

$$
-\Delta \mathcal{V}(x) \geq \varphi_{2}\left(x_{1}, x_{2}\right) \geq \beta\left(\left\|x_{1}\right\|^{2}+\left\|x_{2}\right\|^{2}\right) .
$$

Consider the case $\left\|x_{1}\right\| \geq\left\|x_{2}\right\|$. Then, $\left\|x_{1}\right\|^{2}+\left\|x_{2}\right\|^{2} \geq\left\|x_{1}\right\|^{2} \geq$ $\left(\frac{1}{2}\left\|x_{1}\right\|+\frac{1}{2}\left\|x_{2}\right\|\right)^{2} \geq \frac{1}{4}\|x\|^{2}$. Consider the case $\left\|x_{2}\right\| \geq\left\|x_{1}\right\|$. Then, $\left\|x_{1}\right\|^{2}+\left\|x_{2}\right\|^{2} \geq\left\|x_{2}\right\|^{2} \geq\left(\frac{1}{2}\left\|x_{1}\right\|+\frac{1}{2}\left\|x_{2}\right\|\right)^{2} \geq \frac{1}{4}\|x\|^{2}$. As a consequence, $-\Delta \mathcal{V}(x) \geq \beta\left(\left\|x_{1}\right\|^{2}+\left\|x_{2}\right\|^{2}\right) \geq \frac{\beta}{4}\|x\|^{2}=\alpha_{\Delta}(\|x\|)$, and $\alpha_{\Delta}(\|x\|) \in \mathcal{K}_{\infty}$ which concludes the proof.

Finally, we show that (11), with (10) (or (13)), and (14), achieves (iii) in Problem 1.

Corollary 1: Consider system (7) where $g(\cdot)$ is defined by (11), (10), (14). Let $x(0) \in \mathcal{X}_{f}$, then the closed-loop trajectory is such that there exists a finite $\bar{k} \in \mathbb{Z}_{0+}$ such that $x_{2}(k)=0$, for all $k \geq \bar{k}$.

Proof: By Theorem 5 we have that $\Delta \mathcal{V}(x) \leq-\alpha_{\Delta}(\|x\|)$, $\alpha_{\Delta}(\|x\|) \in \mathcal{K}_{\infty}$. Since $0 \in \operatorname{int}\left(\mathcal{O}_{\infty}^{1}\right)$, there exists $\rho>0$ such that $\left\{x \in \mathbb{R}^{n}:\|x\| \leq \rho\right\} \subseteq \mathcal{O}_{\infty}^{1}$, and $\alpha_{\rho}$ such that $\alpha_{\Delta}(\|x\|) \geq \alpha_{\rho}$, for 
$\|x\|>\rho$. Since $\mathcal{X}_{f}$ is compact, $\|x(0)\|$ is finite, and hence $\mathcal{V}(x(0))$ is finite. Thus, there exists $\bar{k}(x(0))$ such that $\|x(\bar{k}(x(0)))\| \leq \rho$. Assume this is false. Then, $\mathcal{V}(x(k)) \leq \mathcal{V}(x(0))-k \alpha_{\rho}$, and $\lim _{k \rightarrow \infty} \mathcal{V}(x(k))=-\infty$, which contradicts $\mathcal{V}(x) \geq 0$ for all $x \in \mathbb{R}^{n}$. Then, there must exist $\bar{k}(x(0))$ such that $x(\bar{k}(x(0))) \in$ $\left\{x \in \mathbb{R}^{n}:\|x\| \leq \rho\right\} \subseteq \mathcal{O}_{\infty}^{1}$, and hence $x_{1}(\bar{k}(x(0)))=x(\bar{k}(x(0)))$, $x_{2}(\bar{k}(x(0)))=0$. Since cost function (14) is used, $\mathcal{O}_{\infty}^{1}$ is invariant for $A+B_{i} K_{i}$, and $x_{1}(\bar{k}(x(0)))=x(\bar{k}(x(0))), x(k) \in \mathcal{O}_{\infty}^{1}$ for all $k \geq \bar{k}(x(0))$, and thus $x_{2}(k)=0, u_{2}(k)=0$ for all $k \geq \bar{k}(x(0))$.

By summarizing the results of Sections III and IV, the following theorem shows that the proposed VSG solves entirely Problem 1.

Theorem 6: Given (1) subject to constraints (2) and initial state $x_{0} \in \mathcal{X}_{f}$, control strategy (11), (10) (or (13)), (14) solves Problem 1, and ensures that $u_{2} \neq 0$ for a finite time.

Proof: By Theorem 3 (Theorem 4) $\mathcal{X}_{f}$ is PI for (1) in closed loop with (10) (or (13)), (11), and for $x \in \mathcal{X}_{f}, x_{i} \in \mathcal{O}_{\infty}^{i}\left(x_{i} \in \mathcal{O}_{\infty}^{i}\left(\varepsilon_{i}\right)\right)$ and hence (2) is satisfied along the trajectories of the closed-loop system. Hence, $(i)$ holds. By Theorem 5 the closed-loop system is AS, hence (ii) holds. Finally, by using (14) in (10)/(13), if $x(k) \in$ $\mathcal{O}_{\infty}^{1}$, for some $k \in \mathbb{Z}_{0+}, u_{1}(h)=K x(h), u_{2}(h)=0$ for all $h \geq k$. Instead, by the definition of $\mathcal{O}_{\infty}$, if $x(k) \notin \mathcal{O}_{\infty}^{1}$ and $u_{1}(h)=K x(h)$, $u_{2}(h)=0$ for all $h \geq k$, there exists $\bar{k} \geq k$ such that (2) is violated. Thus, (iii) holds. In addition, since $\mathcal{X}_{f}$ is compact, by Corollary 1 , if $x(k) \notin \mathcal{O}_{\infty}^{1}$, there exists a finite $h \in \mathbb{Z}_{0+}$ such that $x(\bar{h}) \in \mathcal{O}_{\infty}^{1}$, and $u_{2}(h)=0$, for all $h \geq \bar{h}$.

\section{Case Study in Attitude Control}

We apply VSG to attitude regulation of a spacecraft equipped with reaction wheels and thrusters. Reaction wheels are powered by solar energy, and hence inexpensive to use, but have small authority. Thrusters have larger authority but consume fuel, so their usage must be minimized. We consider a body fixed frame aligned with the principal axes and with the origin at the satellite center of mass. We call $\vartheta, \varsigma, \psi$ the three Euler angles (roll, pitch, yaw) determining the spacecraft attitude, and $\dot{\vartheta}, \dot{\varsigma}, \dot{\psi}$ the related angular rates. For small angles, the linearized attitude dynamics are described by

$$
\mathbf{J}_{\mathrm{sc}} \dot{\omega}(t)=-\mathbf{J}_{\mathrm{rw}} \alpha(t)+\tau(t),
$$

where $\theta=[\vartheta \varsigma \psi]^{\prime} \in \mathbb{R}^{3}$ is the vector of Euler angles, $\dot{\theta}=\omega=$ $\left[\begin{array}{lll}\dot{\vartheta} & \dot{\zeta} & \dot{\psi}\end{array}\right]^{\prime} \in \mathbb{R}^{3}$ is the vector of angular rates, $\tau=\left[\begin{array}{lll}\tau_{\vartheta} & \tau_{\varsigma} & \tau_{\psi}\end{array}\right]^{\prime} \in \mathbb{R}^{3}$ is the vector of torques by the thrusters, and $\alpha=\left[\begin{array}{lll}\alpha_{\vartheta} & \alpha_{\varsigma} & \alpha_{\psi}\end{array}\right]^{\prime} \in \mathbb{R}^{3}$ is the vector of angular accelerations from the reaction wheels. In (17), $\mathbf{J}_{\mathrm{sc}}, \mathbf{J}_{\mathrm{rw}} \in \mathbb{R}^{3 \times 3}$ are the matrices of the moments of inertia of the spacecraft and of the reaction wheels, respectively. By choosing the principal axes as reference frame and having the reaction wheels aligned with the principal axes, $\mathbf{J}_{\mathrm{sc}}, \mathbf{J}_{\mathrm{rw}}$ are diagonal.

For $x=\left[\begin{array}{ll}\theta^{\prime} & \dot{\theta}^{\prime}\end{array}\right]^{\prime}, u_{1}=\alpha, u_{2}=\tau$, two discrete-time LQR controllers with $T_{s}=1 \mathrm{~s}$ are given for reaction wheels and thrusters, respectively, and cannot be modified. Thus, we implement a VSG to coordinate the thrusters and the reaction wheels, and to enforce constraints. Simulations of the VSG that enforces the actuator constraints $-0.2 \leq\left[u_{1}\right]_{i} \leq 0.2,-0.9 \leq\left[u_{2}\right]_{i} \leq 0.9$, for $i=1,2,3$, are shown in Figure 1, where $\mathcal{V}_{1}\left(x_{1}\right)=x_{1}^{\prime} P_{1} x_{1}, \mathcal{V}_{2}\left(x_{2}\right)=x_{2}^{\prime} P_{2} x_{2}$ and $P_{1}$, $P_{2}$ are the solutions of the Riccati equations for the LQR controllers of reaction wheels and thrusters, respectively. The trajectory in a simulation with initial state $x_{0}=\left[\begin{array}{llllll}0.35 & -0.25 & 0.6 & 0.2 & -0.1 & 0.05\end{array}\right]^{\prime}$ is shown in Figure 1(a), demonstrating that the input constraints are enforced and the thrusters are used only for a (short) finite period. Figure 1(b) shows that along the trajectory from $x_{0}, \mathcal{V}_{1}$ is not monotonically decreasing, but there exists $\gamma>0$, such that $\mathcal{V}(x)=$ $\mathcal{V}_{1}\left(x_{1}\right)+\gamma \mathcal{V}_{2}\left(x_{2}\right)$ is decreasing. In Figure 1(c) the trajectories for
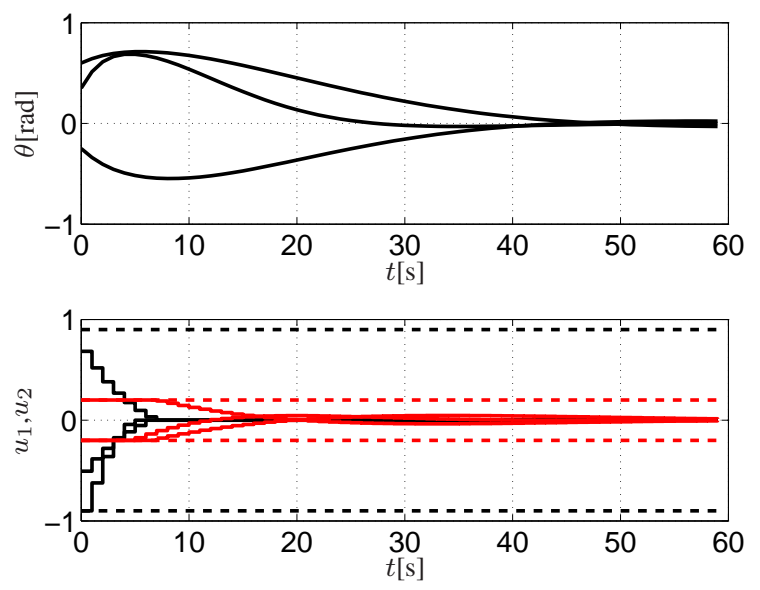

(a) Upper plot: Euler angles. Lower plot: input (solid), and input constraints (dash), $u_{1}$ (red), $u_{2}$ (black).

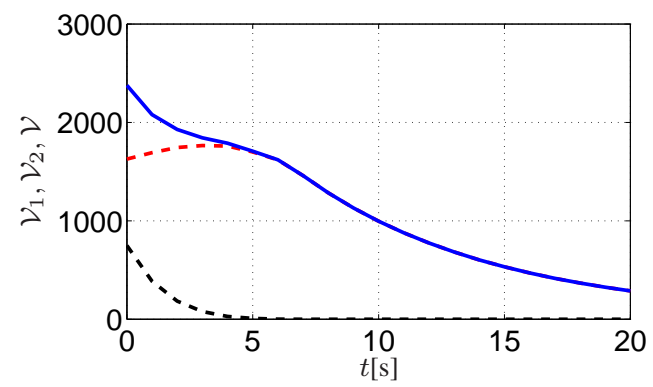

(b) Values of $\mathcal{V}_{1}\left(x_{1}\right)$ (red, dash), $\mathcal{V}_{2}\left(x_{2}\right)$ (black, dash), $\mathcal{V}(x)=\mathcal{V}_{1}\left(x_{1}\right)+\gamma \mathcal{V}_{2}\left(x_{2}\right)$ (blue, solid), for $\gamma=100$.

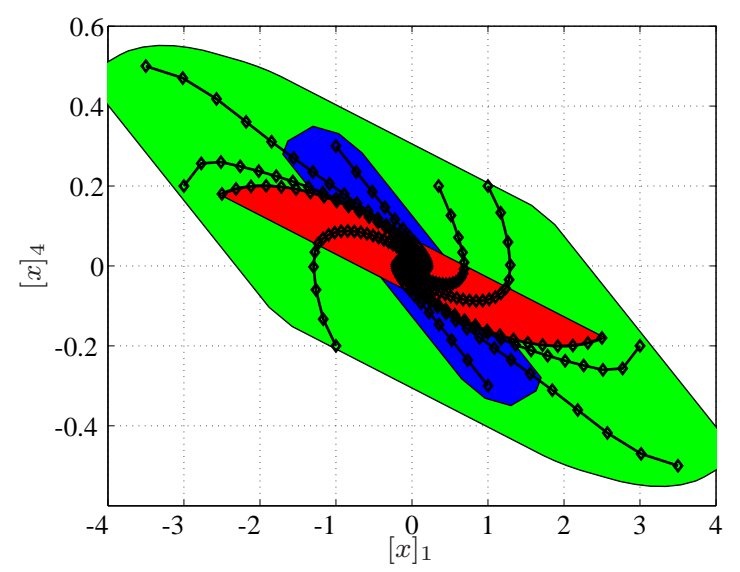

(c) Trajectories in the state plane for different initial conditions. $\operatorname{Proj}]_{[x]_{1,4}}\left[\mathcal{O}_{\infty}^{1}\right]$ (red), $\operatorname{Proj}_{[x]_{1,4}}\left[\mathcal{O}_{\infty}^{2}\right]$ (blue), $\operatorname{Proj}_{[x]_{1,4}}\left[\mathcal{O}_{\infty}^{1} \oplus \mathcal{O}_{\infty}^{2}\right]$ (green).

Figure 1. Simulations of attitude control with input constraints by VSG.

several initial conditions, superimposed to the projections of $\mathcal{O}_{\infty}^{i}$, $i=1,2$ and $\mathcal{O}_{\infty}^{1} \oplus \mathcal{O}_{\infty}^{2}$, on the plane $[x]_{1,4}=\left([x]_{1},[x]_{4}\right)=(\vartheta, \dot{\vartheta})$, are shown, where, by Theorem $3, \mathcal{X}_{f} \equiv\left(\mathcal{O}_{\infty}^{1} \oplus \mathcal{O}_{\infty}^{2}\right)$.

Next, we add the inclusion zone constraint $-0.5 \leq \vartheta \leq 0.5$, which, by defining $y=\vartheta$, is reformulated as output constraints. The trajectory of $\vartheta$ in a simulation with initial state $x_{0}=$

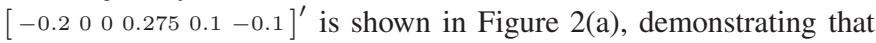
input and output constraints are enforced. In Figure 2(b) the trajectories for several initial conditions are shown, superimposed to the projections of $\overline{\mathcal{O}}_{\infty}^{i}, i=1,2$ and $\overline{\mathcal{O}}_{\infty}^{1} \oplus \overline{\mathcal{O}}_{\infty}^{2}$, on the plane $\left([x]_{1},[x]_{4}\right)$, 
i.e., $(\vartheta, \dot{\vartheta})$, where according to Theorem $4, \mathcal{X}_{f} \subseteq \operatorname{Proj}_{x}\left(\overline{\mathcal{O}}_{\infty}^{1} \oplus \overline{\mathcal{O}}_{\infty}^{2}\right)$.
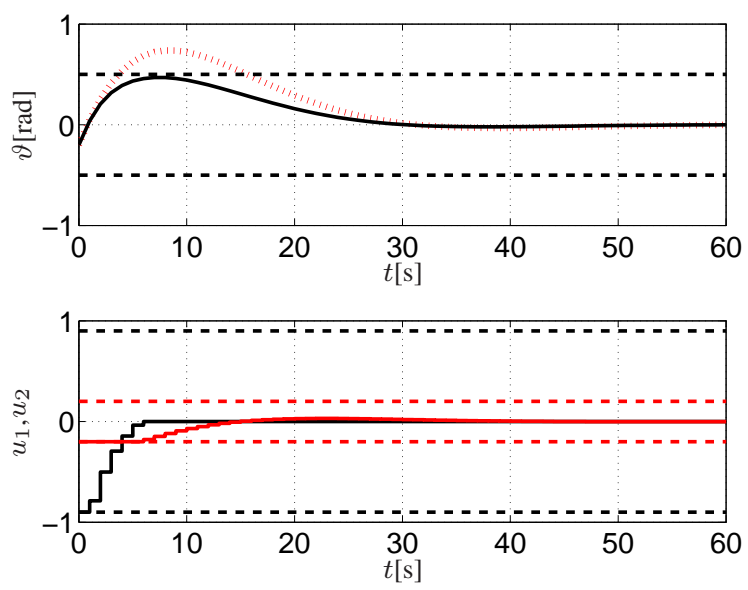

(a) Upper plot: first Euler angle (solid), constraints (dash), and first Euler angle (dot) when $u_{1}=K_{1} x, u_{2}=0$. Lower plot: input (solid), and input constraints (dash), $u_{1}$ (red), $u_{2}$ (black).

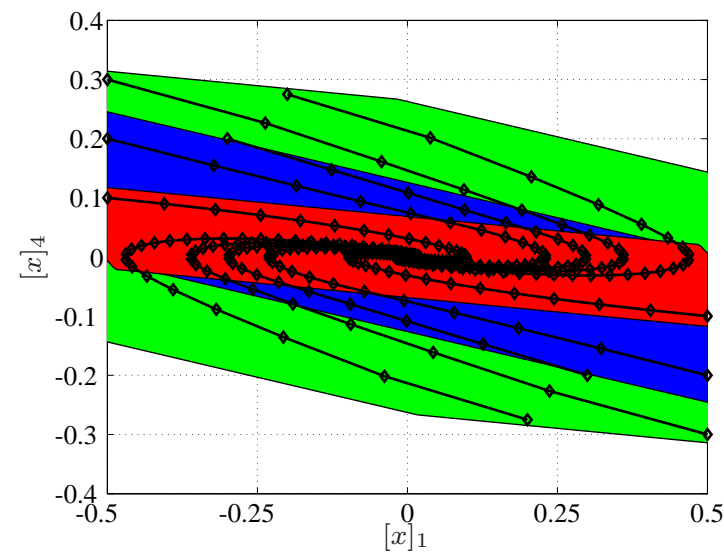

(b) Trajectories in the state plane for different initial conditions. $\operatorname{Proj}_{[x]_{1,4}}\left[\overline{\mathcal{O}}_{\infty}^{1}\right]$ (red), $\operatorname{Proj}_{[x]_{1,4}}\left[\overline{\mathcal{O}}_{\infty}^{2}\right]$ (blue), $\operatorname{Proj}_{[x]_{1,4}}\left[\overline{\mathcal{O}}_{\infty}^{1} \oplus \overline{\mathcal{O}}_{\infty}^{2}\right]$ (green).

Figure 2. Simulations of attitude control with input and output constraints by VSG

The VSG control law as obtained from multiparametric programming consists of 424 regions, reduced to 123 by the merging algorithm in [18]. To further assess the performance, we have compared the VSG with a single (saturated) LQR re-designed to control all actuators. We use as performance metrics the cumulative squared 2-norm of thruster input and state. From the initial conditions as in Figure 2(b), the VSG performance is within $5 \%$ of the single LQR controller tuned to have approximately the same thrusters usage. Thus, even without modifying the existing controllers, VSG achieves a performance close to a full multivariable controller. Furthermore, VSG avoids output constraints violations, while in the simulations from initial conditions as above, the single LQR violates the inclusion zone constraints.

\section{Conclusion And Future Research}

We have proposed a control design for coordinating two groups of actuators, in the case when the controllers for each actuator group are pre-designed, and one group of actuators is "expensive" to operate. We have shown that the resulting closed-loop system satisfies the constraints, is asymptotically stable, and uses the expensive actuators only when otherwise the constraints would be violated, and only for a finite time.

The design can be generalized to the case of $N \in \mathbb{Z}_{+}$actuator groups. At each control cycle, first, the virtual state $x_{N}$ for the most expensive actuator and the cumulative virtual state $x_{(1, \ldots, N-1)}$ for the remaining $N-1$ actuators are generated. Then from $x_{(1, \ldots, N-1)}$, the virtual state $x_{N-1}$ for the second most expensive actuator is obtained, together with $x_{(1, \ldots, N-2)}$, and so on, until all the virtual states have been generated. Note that $N-1$ optimization problems are solved, all with the same number of variables. Hence, the numerical complexity scales linearly with the number of actuator groups. The results of Section IV can be extended to this case with some modifications, for instance due to the fact that the Lyapunov functions are not necessarily quadratic. Future research will explore different splits replacing (10b), the extensions to other classes of preassigned controllers, and to the case of partial state information.

\section{REFERENCES}

[1] S. Di Cairano, D. Yanakiev, A. Bemporad, I. Kolmanovsky, and D. Hrovat, "Model predictive idle speed control: Design, analysis, and experimental evaluation," IEEE Trans. Control Sys. Technology, vol. 20, no. 1, pp. $84-97,2012$.

[2] S. Di Cairano, W. Liang, I. V. Kolmanovsky, M. L. Kuang, and A. M. Phillips, "Power smoothing energy management and its application to a series hybrid powertrain," IEEE Trans. Control Sys. Technology, vol. 21, no. 6, pp. 2091-2103, 2013.

[3] S. Di Cairano, H. Tseng, D. Bernardini, and A. Bemporad, "Vehicle yaw stability control by coordinated active front steering and differential braking in the tire sideslip angles domain," IEEE Trans. Control Sys. Technology, vol. 21, no. 4, pp. 1236-1248, 2013.

[4] J. Buffington, "Tailless aircraft control allocation," in AIAA Guidance, Navigation, and Control Conference and Exhibit, 1997, pp. 737-747.

[5] M. Sidi, Spacecraft dynamics \& control - A practical engineering approach. Cambridge, UK: Cambridge University Press, 1997.

[6] W. Durham, "Constrained control allocation," J. Guidance, Control, and Dynamics, vol. 16, no. 4, pp. 717-725, 1993.

[7] D. Liberzon and A. S. Morse, "Basic problems in stability and design of switched systems," IEEE Control Systems Magazine, vol. 19, no. 5, pp. 59-70, 1999.

[8] S. Di Cairano and I. V. Kolmanovsky, "Constrained actuator coordination by virtual state governing," in Proc. 50th IEEE Conf. on Decision and Control, 2011, pp. 5491-5496.

[9] E. Gilbert and K. Tan, "Linear systems with state and control constraints: the theory and applications of maximal output admissible sets," IEEE Trans. Automatic Control, vol. 36, no. 9, pp. 1008-1020, 1991.

[10] I. Kolmanovsky and E. Gilbert, "Multimode regulators for systems with state and control constraints and disturbance inputs," in Control Using Logic-Based Switching, Springer, 1997.

[11] E. Gilbert and I. Kolmanovsky, "Nonlinear tracking control in the presence of state and control constraints: a generalized reference governor," Automatica, vol. 38, no. 12, pp. 2063-2073, 2002.

[12] A. Bemporad, A. Casavola, and E. Mosca, "Nonlinear control of constrained linear systems via predictive reference management," IEEE Transactions on Automatic Control, vol. 42, pp. 340-349, 1996.

[13] E. G. Gilbert and C.-J. Ong, "Constrained linear systems with hard constraints and disturbances: An extended command governor with large domain of attraction," Automatica, vol. 47, no. 2, pp. 334-340, 2011.

[14] D. Nesic, L. Zaccarian, and A. Teel, "Stability properties of reset systems," Automatica, vol. 44, no. 8, pp. 2019-2026, 2008.

[15] M. Lazar, W. P. M. H. Heemels, and A. Teel, "Lyapunov functions, stability and input-to-state stability subtleties for discrete-time discontinuous systems," IEEE Trans. Automatic Control, vol. 54, no. 10, pp. $2421-2425,2009$.

[16] S. Di Cairano, M. Brand, and S. A. Bortoff, "Projection-free parallel quadratic programming for linear model predictive control," Int. J. Control, vol. 86, no. 8, pp. 1367-1385, 2013.

[17] A. Bemporad, M. Morari, V. Dua, and E. Pistikopoulos, "The explicit linear quadratic regulator for constrained systems," Automatica, vol. 38 , no. 1, pp. 3-20, 2002.

[18] T. Geyer, F. D. Torrisi, and M. Morari, "Optimal complexity reduction of polyhedral piecewise affine systems," Automatica, vol. 44, no. 7, pp. $1728-1740,2008$. 